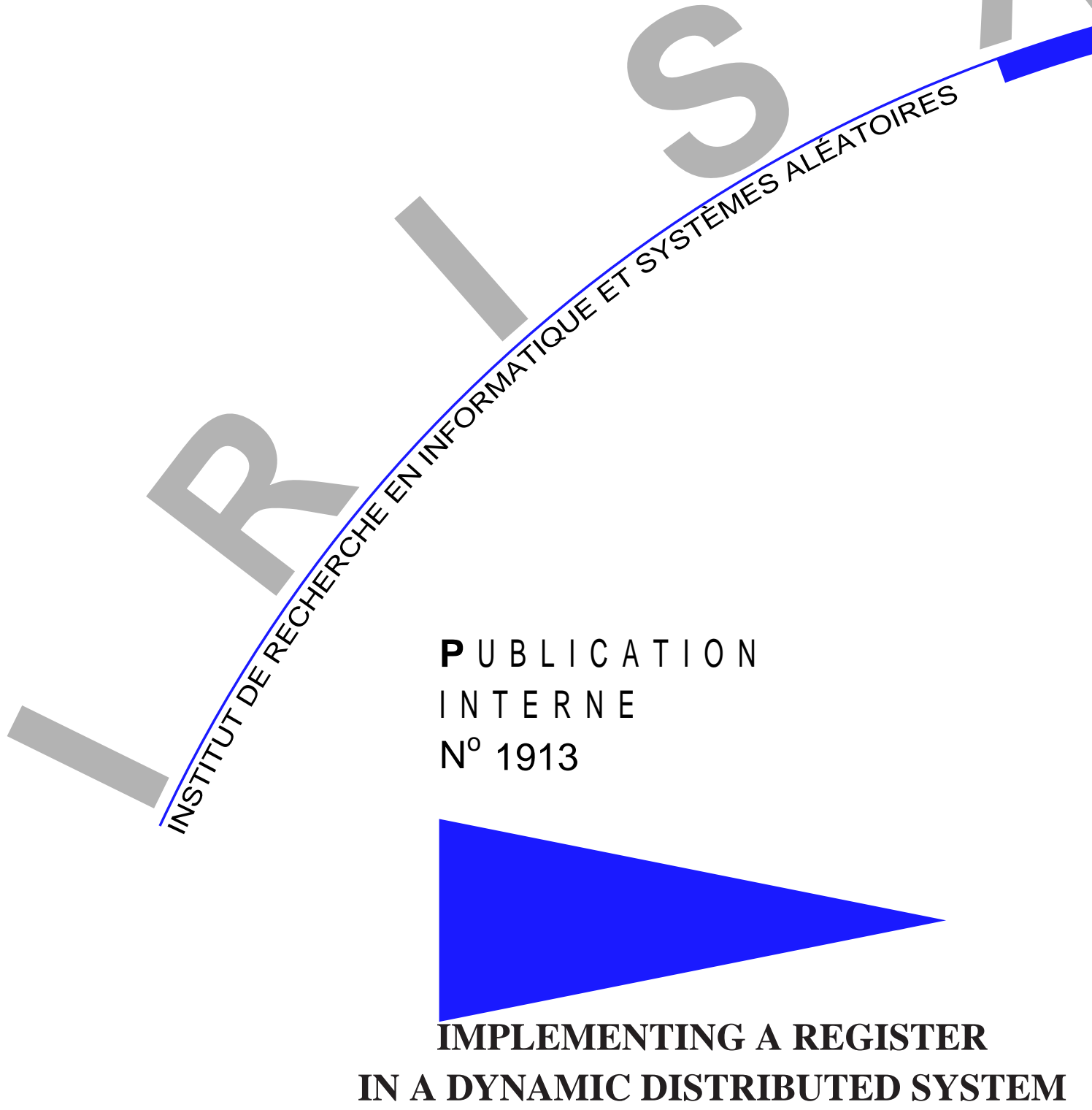

R. BALDONI S. BONOMI A.-M. KERMARREC M. RAYNAL 



\title{
Implementing a Register in a Dynamic Distributed System
}

\author{
R. Baldoni $^{*}$ S. Bonomi $^{* *}$ A.-M. Kermarrec ${ }^{* * *}$ M. Raynal ${ }^{* * * *}$ \\ Systèmes communicants \\ Projet ASAP \\ Publication interne $\mathrm{n}^{\circ} 1913$ - November 2008 - 19 pages
}

\begin{abstract}
Providing distributed processes with concurrent objects is a fundamental service that has to be offered by any distributed system. The classical shared read/write register is one of the most basic ones. Several protocols have been proposed that build an atomic register on top of an asynchronous messagepassing system prone to process crashes. In the same spirit, this paper addresses the implementation of a regular register (a weakened form of an atomic register) in an asynchronous dynamic message-passing system. The aim is here to cope with the net effect of the adversaries that are asynchrony and dynamicity (the fact that processes can enter and leave the system). The paper focuses on the class of dynamic systems the churn rate $c$ of which is constant. It presents two protocols, one applicable to synchronous dynamic message passing systems, the other one to asynchronous dynamic systems. Both protocols rely on an appropriate broadcast communication service (similar to a reliable brodcast). Each requires a specific constraint on the churn rate $c$. Both protocols are first presented in an as intuitive as possible way, and are then proved correct.
\end{abstract}

Key-words: Asynchronous message-passing system, Churn, Dynamic distributed system, Eventually synchronous distributed system, Infinite arrival model, Regular register, Synchronous system.

(Résumé : tsvp)

\footnotetext{
*Universitá La Sapienza, Roma, Italy, Roberto.Baldoni@dis.uniroma1.it

** Universitá La Sapienza, Roma, Italy, Sivia.Bonomi@dis . uniroma1.it

*** INRIA Bretagne Atlantique, Campus de Beaulieu, 35042, Rennes Cedex, France, anne-marie.kermarrec@irisa.fr

**** IRISA, Université de Rennes 1, Campus de Beaulieu, 35042 Rennes Cedex, France, raynal@irisa.fr
}

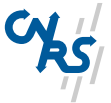

Centre National de la Recherche Scientifique (UMR 6074) Université de Rennes 1 - Insa de Rennes
Institut National de Recherche en Informatique et en Automatique - unité de recherche de Rennes 


\section{Implémentation d'un registre dans un système dynamique}

Résumé : Ce rapport présente deux protocoles qui implémentent un registre régulier dans des systèmes dynamiques respctivement synchone et inéluctablement asynchrone.

Mots clés : Churn, registre régulier, Système asynchrone, Système synchrone. 


\section{Introduction}

On registers A concurrent object is an object that can be accessed by several processes. Among the concurrent objects, a register is certainly one of the most basic ones. A register provides the processes with two operations one that allows them to read the value of the object, the other one that allows them to define the new value of the object. According to the value domain of the register, the set of processes that are allowed to read it, the ones that are allowed to write it, and the specification of which value is the value returned by a read operation, a family of types of registers can be defined.

As far as the last point (the value returned by a read operation) is concerned, Lamport has defined three types of register [20].

- a safe register can be written by one writer only. Moreover, a read operation on such a register returns its current value if no write operation is concurrent with that read. In case of concurrency, the read can return any value of the value domain of the register (which means that a read concurrent with a write can return a value that has never been written). This type of register is very weak.

- A regular register can have any number of writers and any number of readers. The writes appear as if they were executed sequentially, this sequence complying with their real time order (i.e., if two writes $w_{1}$ and $w_{2}$ are concurrent they can appear in any order, but if $w_{1}$ terminates before $w_{2}$ starts, $w_{1}$ has to appear as being executed before $w_{2}$ ).

As far as a read operation is concerned we have the following. If no write operation is concurrent with a read operation, that read operation returns the current value kept in the register. Otherwise, the read operation returns any value written by a concurrent write operation of the last value of the register before these concurrent writes. A regular register is stronger than a safe register, as the value returned in presence of concurrent write operations is no longer arbitrary.

Nevertheless, a regular register can exhibit what is called a new/old inversion. The figure on the right depicts two write operations $w_{1}$ and $w_{2}$ and two read operations $r_{1}$ and $r_{2}$ that are concurrent ( $r_{1}$ is concurrent

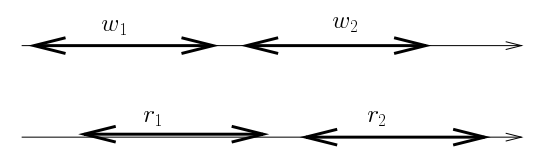
with $w_{1}$ and $w_{2}$, while $r_{2}$ is concurrent with $w_{2}$ only). According to the definition of register regularity, it is possible that $r_{1}$ returns the value written by $w_{2}$ while $r_{2}$ returns the value written by $w_{1}$.

- An atomic register is a regular register without new/old inversion. This means that an atomic register is such that all its read and write operations appear as if they have been executed sequentially, this sequential total order respecting the real time order of the operations. (Linearizability [18] is nothing else than atomicity when we consider objects defined by a sequential specification).

Interestingly enough, safe, regular and atomic registers have the same computational power. This means that it is possible to implement a multi-writer/multi-reader atomic register from single-writer/single-reader safe registers. There is a huge number of papers in the literature discussing such transformations (e.g., $[5,7,16,21,27,29,30]$ to cite a few).

On message-passing dynamic distributed systems The advent of new classes of applications (social networks, security, smart objects sharing etc) and technologies (VANET, WiMax, Airborn Networks, DoD Global Information Grid, P2P) are radically changing the way in which distributed systems are perceived. Such emerging systems have a composition, in term processes participating to the system, that is self-defined at run time depending, for example, on their will to belong to such a system, on the geographical distribution of processes etc. Therefore one of the common denominators of such emerging systems is the dynamicity dimension that introduces a new source of unpredictability inside a distributed system.

PI $n{ }^{\circ} 1913$ 
As a consequence, any specification of a distributed computing abstraction (e.g., registers, communication, consensus) has to take this dynamicity into account and protocols implementing them have to be correct despite the fact that processes enter and leave the system at will. This dynamicity makes abstractions more difficult to understand and master than in traditional distributed systems in which a system starts and remains forever with the same set of processes. The churn notion has been introduced as a system parameter that aims at capturing this dynamicity and makes it tractable by distributed protocols (e.g., [19, 22, 24]). Although numerous protocols have been designed for dynamic distributed message-passing systems, very few papers (such as $[2,4,28]$ ) strive to present models suited to such systems, and extremely few dynamic protocols have been proved correct. Up to now, the most common approach used to address dynamic systems is mainly experimental.

Contribution and roadmap This paper addresses the implementation of a regular register abstraction in a synchronous and eventually synchronous message-passing distributed system subject to a constant churn. We focus on regular registers as they have the same computability power as the atomic registers but are easier to implement in a traditional distributed system [3]. Moreover, interestingly enough, regular registers allow solving some basic coordination problems such as the consensus problem in asynchronous systems prone to crash but equipped with an appropriate leader election service [11,14]. Therefore this paper makes a step in giving a clear specification of the abstraction of regular register in a dynamic context and provides two formally correct implementations of the specification.

To that end, Section 2 first introduces base definitions. Then, the paper considers three type of dynamic systems, namely synchronous dynamic systems (Section 3), fully asynchronous dynamic systems (Section 4), and eventually synchronous dynamic systems (Section 5). It presents two protocols that build a regular register (in synchronous and eventually synchronous systems) and shows that it is impossible to build a regular register in a fully asynchronous dynamic system. The dynamicity attribute of the three models is defined from the churn parameter.

\section{Base definitions}

\subsection{Dynamic system}

The processes In a dynamic system, entities may join and leave the system at will. Consequently, at any point in time, the system is composed of the processes (nodes) that have joined and have not yet left the system. In order to model processes continuously arriving to and departing from the system, we assume the infinite arrival model (as defined in [25]). In each run, infinitely many processes $\Pi=\left\{\ldots, p_{k}, p_{j}, p_{k}, \ldots\right\}$ may a priori join the system, but at any point in time the number of processes is bounded. Processes are sequential processing entities (sometimes called nodes). Moreover, we assume that the processes are uniquely identified (with their indexes).

Time model The underlying time model is the set of positive integers.

Entering and leaving the system When a process $p_{i}$ enters the system it executes the operation join(). That operation, invoked at some time $\tau$, is not instantaneous: it consumes time. But, from time $\tau$, the process $p$ can receive and process messages sent by any other process that belongs to the system.

To explicit the "begin of a join()" notion (time $\tau$ ), let us consider the case of mobile nodes in a wireless network. The beginning of its join() occurs when a process (node) enters the geographical zone within which it can receive messages. The end of the join() depends on the code associated with that operation. The important point is that the process $p$ is in the listening mode from the beginning of join(). If the code of 
the join() operation contains waiting periods, the process $p$ can receive and process messages during these waiting periods. So, a process is in the listening mode since the invocation of join(), and proceeds to the active mode only when the join() operation terminates. It remains in the active mode until it leaves the system.

A process leaves the system in an implicit way. When it does, it leaves the system forever and does not longer send or receive messages. From a practical point of view, if a process wants to re-enter the system, it has to enter it as a new process (i.e., with a new name). Let us observe that, while, from the application point of view, a crash is an involuntary departure, there is no difference with a voluntary leave from the model point of view. So, considering a crash as an unplanned leave, the model can take them into account without additional assumption.

Definition 1 A process is active from the time it returns from the join() operation until the time it leaves the system. $A(\tau)$ denotes the set of processes that are active at time $\tau$, while $A\left[\tau_{1}, \tau_{2}\right]$ denotes the set of processes that are active during the time interval $\left[\tau_{1}: \tau_{2}\right]$ (hence, $A(\tau)=A[\tau, \tau]$ ).

Churn rate The dynamicity of the joins and leaves of the processes is captured by the system parameter called churn. As in a lot of other papers we consider here the churn rate, denoted $c$, defined as the percentage of the nodes that are "refreshed" at every time unit $(c \in[0,1])$. This means that, while the number of processes remains constant (equal to $n$ ), in every time unit $c \times n$ processes leave the system and the same number of processes join the system. It is shown in [19] that this assumption is fairly realistic for several classes of applications built on top of dynamic systems.

\subsection{Regular register}

The notion of a regular register defined in the introduction has to be adapted to a dynamic system. We consider that a protocol implements a regular register in a dynamic system if the following properties are satisfied.

- Liveness: If a process invokes a read or a write operation and does not leave the system, it eventually returns from that operation.

- Safety: A read operation returns the last value written before the read invocation, or a value written by a write operation concurrent with it.

It is easy to see that these properties boil down to the classical definition if the system is static. Moreover, it is assumed that a process invokes the read or write operation only after it has returned from its join() invocation.

\section{Regular register in a synchronous system}

\subsection{Assumptions}

This section presents a protocol that implements a one-writer/multi-reader regular register in a dynamic system where the churn rate $c$ is constant, the number $n$ is known by every process, and the processes can access a global clock $^{2}$. The protocol assumes that the churn rate is such that $c<1 /(3 \delta)$ (where $\delta$ is a bound on communication delays defined below). Let us observe that, while relating $c$ to $\delta$, this constraint is independent of the system size $n$ (as we will see, this will be different for eventually synchronous systems).

\footnotetext{
${ }^{1}$ Actually, the protocol works for any number of writers as long as the writes are not concurrent. Considering a single writer makes the exposition easier.

${ }^{2}$ The global clock is for ease of presentation. As we are in a synchronous system, this global clock can be implemented by synchronized local clocks.

PI ${ }^{\circ} 1913$
} 


\subsection{Synchronous system}

The system is synchronous in the following sense. The processing times of local computations (but the wait statement) are negligible with respect to communication delays, so they are assumed to be equal to 0 . Contrarily, messages take time to travel to their destination processes.

Point-to-point communication The network allows a process $p_{i}$ to send a message to another process $p_{j}$ as soon as $p_{i}$ knows that $p_{j}$ has entered the system. The network is reliable in the sense that it does not loose, create or modify messages. Moreover, the synchrony assumption guarantees that if $p$ invokes "send $m$ to $p_{j}$ " at time $\tau$, then $p_{j}$ receives that message by time $\tau+\delta^{\prime}$ (if it has not left the system by that time). In that case, the message is said to be "sent" and "received".

Broadcast It is assumed that the system is equipped with an appropriate broadcast communication subsystem that provides the processes with two operations, denoted broadcast() and deliver(). The former allows a process to send a message to all the processes in the system, while the latter allows a process to deliver a message. Consequently, we say that such a message is "broadcast" and "delivered". These operations satisfy the following property.

- Timely delivery: Let $\tau$ be the time at which a process $p$ invokes broadcast $(m)$. There is a constant $\delta$ $\left(\delta \geq \delta^{\prime}\right)$ (known by the processes) such that if $p$ does not leave the system by time $\tau+\delta$, then all the processes that are in the system at time $\tau$ and do not leave by time $\tau+\delta$, deliver $m$ by time $\tau+\delta$.

Such a pair of broadcast operations has first been formalized in [15] in the context of systems where process can commit crash failures. It has been extended to the context of dynamic systems in [10].

\subsection{A protocol for synchronous dynamic systems}

The principle that underlies the design of the protocol is to have fast reads operations: a process willing to read has to do it locally. From an operational point of view, this means that a read is not allowed to use a wait() statement, or to send messages and wait for associated responses. Hence, albeit the proposed protocol works in all cases, it is targeted for applications where the number of reads outperforms the number of writes.

Local variables at a process $p_{i} \quad$ Each process $p_{i}$ has the following local variables.

- Two variables denoted register $_{i}$ and $s n_{i}$; register $_{i}$ contains the local copy of the regular register, while $s n_{i}$ is the associated sequence number.

- A boolean active $_{i}$, initialized to false, that is switched to true just after $p_{i}$ has joined the system.

- Two set variables, denoted replies $i$ and reply_to $_{i}$, that are used during the period during which $p_{i}$ joins the system. The local variable replies $_{i}$ contains the 3 -uples $\langle$ id, value, sn $\rangle$ that $p_{i}$ has received from other processes during its join period, while reply_to contains the processes that are joining the system concurrently with $p_{i}$ (as far as $p_{i}$ knows).

Initially, $n$ processes compose the system. The local variables of each of these processes $p_{k}$ are such that register $_{k}$ contains the initial value of the regular register ${ }^{3}, s n_{k}=0$, active $_{k}=$ true $_{\text {, and }}$ replies $_{k}=$ reply_to ${ }_{k}=\emptyset$.

\footnotetext{
${ }^{3}$ Without loss of generality, we assume that at the beginning every process $p_{k}$ has in its variable register $_{k}$ the value 0
} 
The join() operation When a process $p_{i}$ enters the system, it first invokes the join operation. The algorithm implementing that operation, described in Figure 1, involves all the processes that are currently present (be them active or not).

First $p_{i}$ initializes its local variables (line 01), and waits for a period of $\delta$ time units (line 02); This waiting period is explained later. If register $i$ has not been updated during this waiting period (line 03), $p$ broadcasts (with the broadcast () operation) an INQUIRY $(i)$ message to the processes that are in the system (line 05) and waits for $2 \delta$ time units, i.e., the maximum round trip delay (line 06$)^{4}$. When this period terminates, $p_{i}$ updates its local variables register $i$ and $s n_{i}$ to the most uptodate values it has received (lines 07-08). Then, $p_{i}$ becomes active (line 10), which means that it can answer the inquiries it has received from other processes, and does it if reply_to $\neq \emptyset$ (line 11). Finally, $p_{i}$ returns $o k$ to indicate the end of the join () operation (line 12).

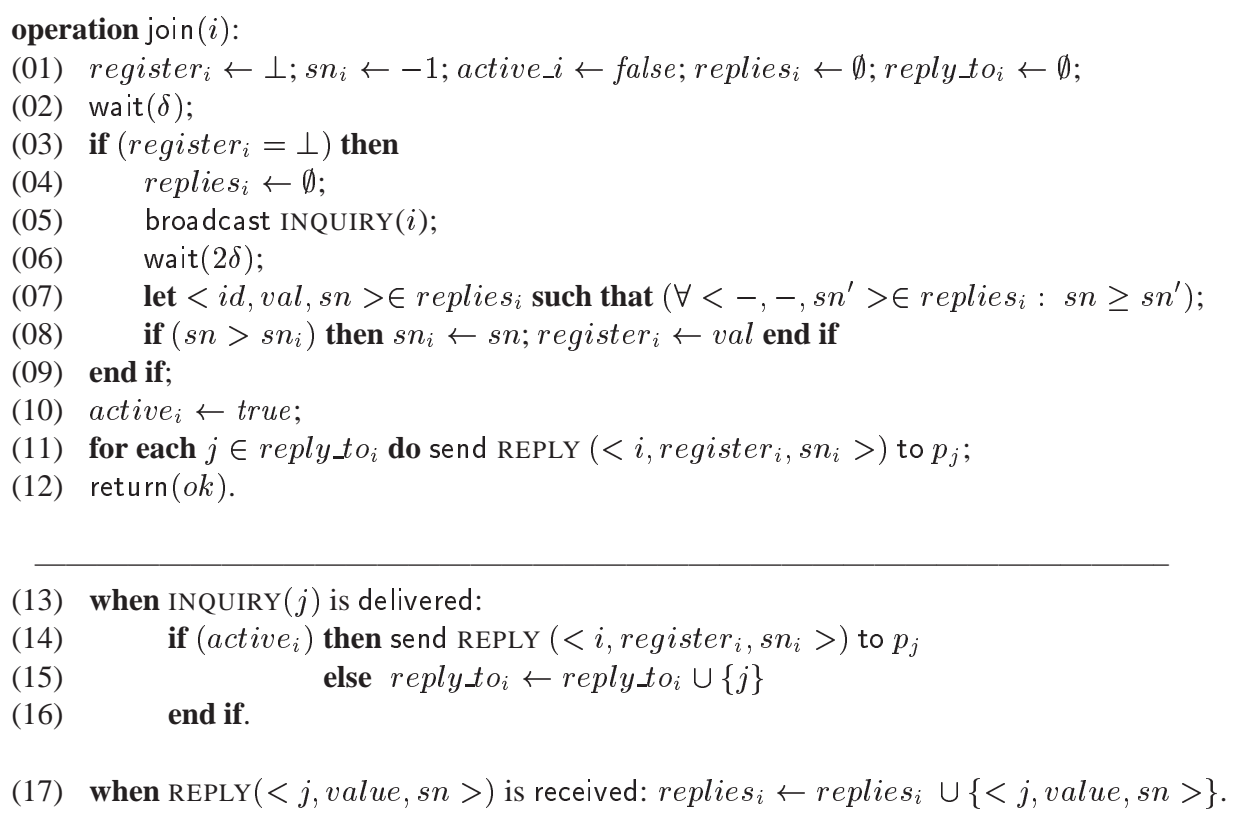

Figure 1: The join() protocol for a synchronous system (code for $p_{\imath}$ )

When a process $p_{i}$ receives a message $\operatorname{INQUIRY}(j)$, it answers $p_{j}$ by return sending back a REPLY $(<$ $i$, register,$s n_{i}>$ ) message containing its local variable if it is active (line 14). Otherwise, $p$ postpones its answer until it becomes active (line 15 and lines 10-11). Finally, when $p_{\imath}$ receives a message REPLY $(<$

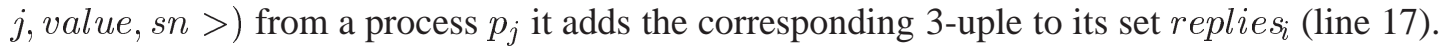

The $\operatorname{read}()$ and write $(v)$ operations The algorithms for the read and write operations associated with the regular register are described in Figure 2. The read is purely local (i.e., fast): it consists in returning the current value of the local variable register . $_{\text {. }}$

\footnotetext{
${ }^{4}$ The statement wait $(2 \delta)$ can be replaced by wait $\left(\delta+\delta^{\prime}\right)$, which provides a more efficient join operation; $\delta$ is the upper bound for the dissemination of the message sent by the reliable broadcast that is a one-to-many communication primitive, while $\delta^{\prime}$ is the upper bound for a response that is sent to a process whose id is known, using a one-to-one communication primitive. So, wait $(\delta)$ is related to the broadcast, while wait $\left(\delta^{\prime}\right)$ is related to point-to-point communication. We use the wait $(2 \delta)$ statement to make easier the presentation.
}

PI ${ }^{\circ} 1913$ 
The write consists in disseminating the new value $v$ (together with its sequence number) to all the processes that are currently in the system (line 01). In order to guarantee the correct delivery of that value, the writer is required to wait for $\delta$ time units before terminating the write operation (line 02 ).

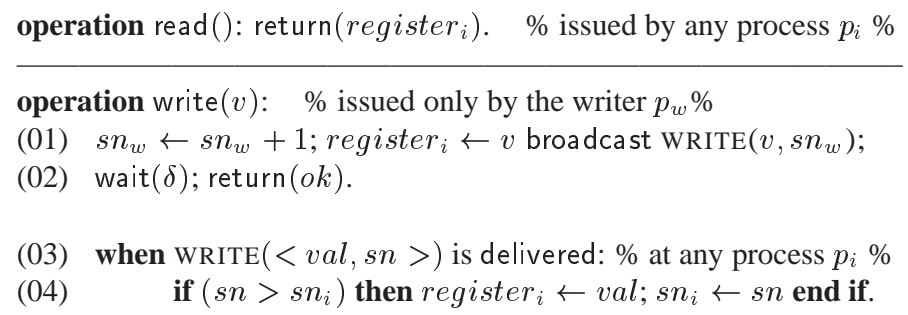

Figure 2: The read () and write() protocols for a synchronous system

Why the wait $(\delta)$ statement at line 02 of the join( $)$ operation? To motivate the wait $(\delta)$ statement at line 02, let us consider the execution of the join() operation depicted in Figure 3(a). At time $\tau$, the processes $p_{j}, p_{h}$ and $p_{k}$ are the three processes composing the system, and $p_{j}$ is the writer. Moreover, the process $p_{i}$ executes join() just after $\tau$. The value of the copies of the regular register is 0 (square on the left of $p, p_{h}$ and $p_{k}$ ), while register $i=\perp$ (square on its left). The "timely delivery" property of the broadcast invoked

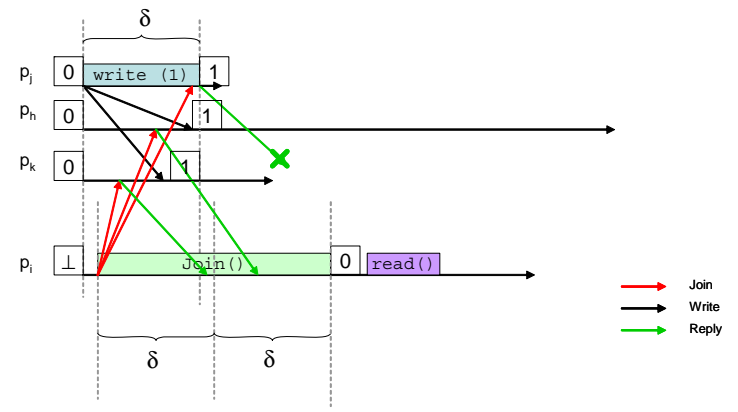

(a) Without wait $(\delta)$

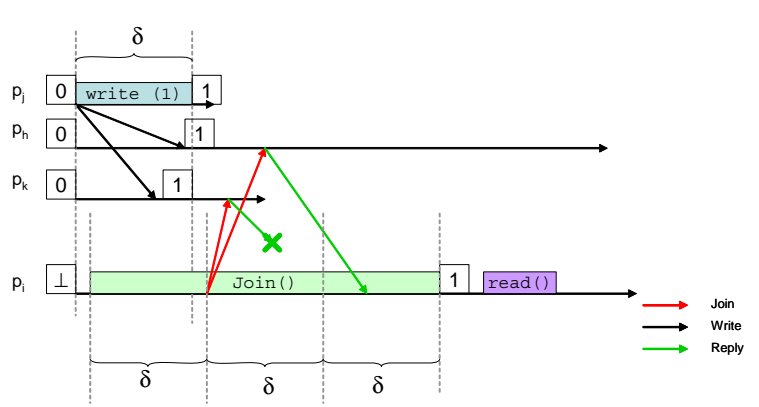

(b) With wait $(\delta)$

Figure 3: Why wait $(\delta)$ is required

by the writer $p_{j}$ ensures that $p_{j}$ and $p_{k}$ deliver the new value $v=1$ by $\tau+\delta$. But, as it entered the system after $\tau$, there is no such a guarantee for $p_{i}$. Hence, if $p_{i}$ does not execute the wait $(\delta)$ statement at line 02 , its execution of the lines 03-09 can provide it with the previous value of the regular register, namely 0 . If after obtaining $0, p_{i}$ issues another read it obtains again 0 , while it should obtain the new value $v=1$ (because 1 is the last value written and there is no write concurrent with this second read issued by $p$ ).

The execution depicted in Figure 3(b) shows that this incorrect scenario cannot occur if $p$ is forced to wait for $\delta$ time units before inquiring to obtain the last value of the regular register. 


\subsection{Proof of the synchronous protocol}

Lemma 1 Termination. If a process invokes the join() operation and does not leave the system for at least $3 \delta$ time units, or invokes the read() operation, or invokes the write () operation and does not leave the system for at least $\delta$ time units, it does terminates the invoked operation.

Proof The read() operation trivially terminates. The termination of the join () and write() operations follows from the fact that the wait() statement terminates.

Let us recall that $A[\tau, \tau+x]$ denotes the set of processes that are active (at least) during the period $[\tau, \tau+x]$.

Lemma 2 Let $c<1 / 3 \delta . \forall \tau:|A[\tau, \tau+3 \delta]| \geq n(1-3 \delta c)>0$.

Proof Let us first consider the case $\tau_{0}=0$. We have $\left|A\left(\tau_{0}\right)\right|=n$. Then, due to definition of $c$, we have $\left|A\left[\tau_{0}, \tau_{0}+1\right]\right|=n-n c$. During the second time unit, $n c$ new processes enter the system and replace the $n c$ processes that left the system during that time unit. In the worst case, the $n c$ processes that left the system are processes that were present at time $\tau_{0}$ (i.e., they are not processes that entered the system between $\tau_{0}$ and $\left.\tau_{0}+1\right)$. So, we have $\left|A\left[\tau_{0}, \tau_{0}+2\right]\right| \geq n-2 n c$. If we consider a period of $3 \delta$ time units, i.e. the longest period needed to terminate a join operation, we obtain $\left|A\left[\tau_{0}, \tau_{0}+3 \delta\right]\right| \geq n-3 \delta n c=n(1-3 \delta c)$. Moreover, as $c<1 / 3 \delta$, we have $|A[\tau, \tau+3 \delta]| \geq n(1-3 \delta c)>0$.

It is easy to see that the previous reasoning depends only on (1) the fact that there are $n$ processes at each time $\tau$, and (2) the definition of the churn rate $c$, from which we conclude that $\forall \tau:|A[\tau, \tau+3 \delta]| \geq$ $n(1-3 \delta c)$.

$\square$ Lemma 2

Lemma 3 Let $c<1 / 3 \delta$. When a process $p_{i}$ terminates the execution of join(), its local variable register contains the last value written in the regular register (i.e., the last value before the join() invocation), or a value whose write is concurrent with the join() operation.

Proof Let $p_{i}$ be a process that issues a join() operation. It always executes the wait $(\delta)$ statement at line 02 . Then, there are two cases according to the value of the predicate register $_{i}=\perp$ evaluated at line 03 of the join operation.

- Case register $_{i} \neq \perp$. We then conclude that $p_{i}$ has received a WRITE $(<$ val, sn $>)$ message and accordingly updated register $_{i}$ line 04 , Figure 2 . As (1) the write operation lasts $\delta$ time units (line 02 of the write operation, Figure 2), (2) the join operation lasts at least $\delta$ time units, and (3) the message $\operatorname{WRITE}(<v a l, s n>)$ - sent at the beginning of the write - takes

at most $\delta$ time units, it follows from register $i \neq \perp$ that the join() and the write() operations overlap, i.e., they are concurrent, which proves the lemma for that case. (The worst case scenario is depicted on the right.)

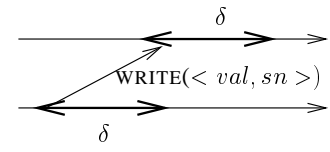

- register $_{i}=\perp$. In that case, $p_{i}$ broadcasts an $\operatorname{INQUIRY}(i)$ message and waits for $2 \delta$ time units (lines 05-06 of the join() operation, Figure 1). Let $\tau$ be the time at with $p_{i}$ broadcasts the INQUIRY $(i)$ message. At the end of the $2 \delta$ round trip upper bound delay, $p_{i}$ updates register $_{i}$ with the value associated with the highest sequence number it has received (lines 07-08). We consider two subcases.

- No write is concurrent with the join operation. As $|A[\tau, \tau+3 \delta]| \geq n(1-3 \delta c)>0$ (Lemma 2), $A[\tau, \tau+3 \delta]$ is not empty. Consequently, at least one process that has a copy of the last written value answers the inquiry of $p_{i}$ and consequently $p_{i}$ sets register $i$ to that value by $2 \delta$ time units after the broadcast, which proves the lemma.

PI ${ }^{\circ} 1913$ 
- There is (at least) one write issued by a process $p_{j}$ concurrent with the join operation. In that case, $p_{i}$ can receive both WRITE $(<v a l, s n>)$ messages and REPLY $(<j$, val, sn $>)$ messages. According the values received at time $\tau+2 \delta, p_{i}$ will update register $_{i}$ to the value written by a concurrent update, or the value written before the concurrent writes.

$\square$ Lemma 3

Lemma 4 Safety. Let $c<1 / 3 \delta$. A read() operation returns the last value written before the read invocation, or a value written by a write operation concurrent with it.

Proof Let us observe that a read by any process can be invoked only after that process has terminated its join operation. It follows from that observation, Lemma 3, and the lines 03-04 associated with the write operation (Figure 2) that a read always returns a value that has been written.

Let us observe that a write that starts at time $\tau$, terminates at time $\tau+\delta$, and all the processes in $A[\tau, \tau+\delta]$ have delivered the value it has written by $\tau+\delta$. Considering that observation, the proof that a read operation returns the last written value or a value written by a concurrent write is similar to the proof of the previous lemma. It is left to the reader.

$\square_{\text {Lemma } 4}$

Theorem 1 Let $c<1 / 3 \delta$. The protocol described is the Figures 1 and 2 implements a regular register in a synchronous dynamic system.

Proof The proof follows directly from Lemmas 1 and 4.

$\square$ Theorem 1

\section{Regular register in an asynchronous system}

This section shows that (not surprisingly) it is not possible to implement a regular register in a fully asynchronous dynamic system. Such a system is similar to the synchronous system defined in Section 3 with the following difference: there are no bound on message transfer delays such as $\delta$ and $\delta$.

Theorem 2 It is not possible to implement a regular register in a fully asynchronous dynamic system.

Proof The proof is a consequence of the impossibility to implement a register in a fully asynchronous static message-passing system prone to any number of process crashes [3 5 . More specifically, if processes enter and leave the system, due to the absence of an upper bound on message transfer delays, it is easy possible a run in which the value obtained by a process is always older than the last value whose write is terminated.

$\square$ Theorem 2

\section{Regular register in an eventually synchronous system}

\subsection{System model}

In static systems, an eventually synchronous distributed system ${ }^{6}$ is a system that after an unknown but finite time behaves synchronously [6,9]. We adopt the same definition for dynamic systems. More precisely:

\footnotetext{
${ }^{5}$ This paper shows also that such a construction is possible as soon as a majority of processes do not crash.

${ }^{6}$ Sometime also called partially synchronous system.
} 
- As in an asynchronous system, there is a time notion (whose domain is the set of integers), but this time notion is inaccessible to the processes. The definition of the churn rate $c$ is the same as in Section 2.1 , i.e., the rate of the number of processes that join/leave the system per time unit.

- Eventual timley delivery: There is a time $\tau$ and a bound $\delta$ such that any message sent (broadcast) at time $\tau^{\prime} \geq \tau$, is received (delivered) by time $\tau^{\prime}+\delta$ to the processes that are in the system during the interval $\left[\tau^{\prime}, \tau^{\prime}+\delta\right]$.

It is important to observe that the date $\tau$ and the bound $\delta$ do exist but can never be explicitly known by the processes.

\subsection{Assumptions}

The proposed protocol is based on the following assumptions.

- $\forall \tau:|A(\tau)|>\frac{n}{2}$. This assumption states that, at any time $\tau$, a majority of the $n$ processes that currently compose the system are active. Let us recall that a process becomes active as soon as it has obtained a copy of the regular register. This assumption is similar to the "majority of non-faulty processes" assumption used in the design of fault-tolerant protocols suited to asynchronous systems prone to process crashes. The "spirit" of these assumptions is the same: the majority of active (resp., non-faulty) processes is needed to preserve the consistency of the regular register (resp., system state).

- $c<1 /(3 \delta n)$. This assumption restricts the churn rate that defines the dynamicity of the system. Differently from synchronous systems, it involves both the eventual bound $\delta$ and the size $n$ of the system.

\subsection{A protocol for eventually synchronous dynamic system}

As it cannot rely on the passage of time combined with a safe known bound on message transfer delay, the protocol is based on acknowledgment messages. Moreover, it allows any process to write under the assumption that no two processes write concurrently (this assumption could be implemented with appropriate quorums, but we do not consider its implementation in this paper).

Local variables at a process $p_{i} \quad$ Each process $p_{i}$ has to manage local variables, where (as before) register $r_{i}$ denotes the local copy of the regular register. In order to ease the understanding and the presentation of the protocol, the control variables that have the same meaning as in the synchronous protocol are denoted with the same identifiers (but their management can differ). Those are the variables denoted sn $_{t}$, active $_{i}$, replies $_{i}$ and reply_to ${ }_{i}$ in Section 3. In addition to these control variables, the protocol uses the following ones.

- read_sn $n_{i}$ is a sequence number used by $p_{i}$ to distinguish its successive read requests. The particular value read_sn $n_{i}=0$ is used by the join operation.

- reading $_{i}$ is boolean whose value is true when $p_{i}$ is reading.

- write_ack $k_{i}$ is a set used by $p_{i}$ (when it writes a new value) to remember the processes that have acknowledged its last write.

- $d l_{-}$prev $_{i}$ is a set where (while it is joining the system) $p_{i}$ records the processes that have acknowledged its inquiry message while they were not yet active (so, these processes were joining the system too) or while they are reading. When it terminates its join operation, $p_{\imath}$ has to send them a reply to prevent them to be blocked forever.

PI ${ }^{\circ} 1913$ 
Remark In the following, the processing associated with a message reception (if the message has been sent) or a message delivery (if the message has been broadcast) appears in the description of one of the operations join(), read(), or write(). But the sending (or broadcast) of the message that triggers this processing is not necessarily restricted to that operation.

The join() operation The algorithm implementing this operation is described in Figure 4. (The read algorithm -Figure 5- can be seen as a simplified version of it.)

After having initialized its local variables, the process $p_{i}$ broadcasts an INQUIRY $\left(i, r e a d_{\_} s n_{i}\right)$ message to inform the other processes that it enters the system and wants to obtain the value of the regular register (line 03, as indicated read_sn is then equal to 0). Then, after it has received "enough" replies (line 04), $p_{i}$ proceeds as in the synchronous protocol: it updates its local pair $\left(\right.$ register $\left._{i}, s n_{i}\right)$ (lines 05-06), becomes active (line 07), and sends a reply to the processes in the set reply ta (line 08-10). It sends such a reply message also to the processes in its set $d l_{-} p r e v_{i}$ to prevents them from waiting forever. In addition to the tern $<i$, register $_{i}, s n_{i}>$, a reply message sent to a process $p_{j}$, from a process $p_{i}$, has now to carry also the read sequence number $r_{-} s n$ that identifies the corresponding request issued by $p_{j}$.

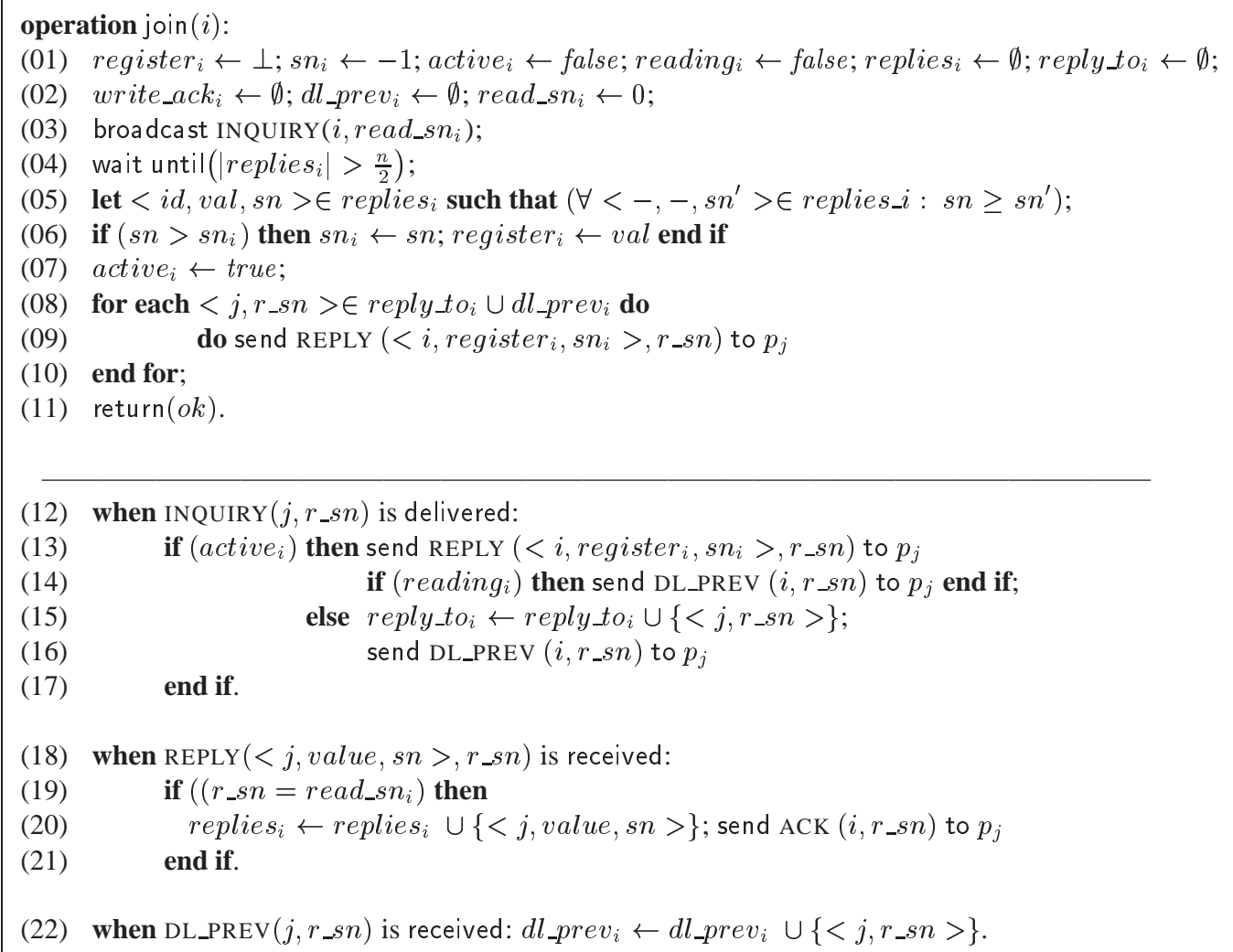

Figure 4: The join() protocol for an eventually synchronous system (code for $p$ )

The behavior of $p_{i}$ when it receives an INQUIRY $\left(j, r_{-} s n\right)$ message is similar to one of the synchronous protocol, with two differences. The first is that it always sends back a message to $p_{j}$. It sends a REPLY() message if it is active (line 13), and a DLPREV() if it not active yet (line 16). The second difference is that, if $p_{i}$ is reading, it also sends a DL_PREV() message to $p_{j}$ (line 14); this is required to have $p_{j}$ send to $p_{i}$ the value it has obtained when it terminates its join operation. 
When $p_{i}$ receives a REPLY $\left(<j\right.$, value, $\left.s n>, r_{-} s n\right)$ message from a process $p_{j}$, if the reply message is an answer to its INQUIRY (i,read_sn) message (line19), $p_{i}$ adds $<j$, value, sn $>$ to the set of replies it has received so far and sends back an $\operatorname{ACK}\left(i, r_{-} s n\right)$ message to $p_{j}$ (line 20).

Finally, when $p_{i}$ receives a message DL_PREV $\left(j, r_{-} s n\right)$, it adds its content to the set $d l_{-} p r e v_{i}$ (line 22), in order to remember that it has to send a reply to $p_{j}$ when it will become active (lines 08-09).

The $\operatorname{read}()$ operation The read() and join() operations are strongly related. More specifically, a read is a simplified version of the join operation ${ }^{7}$. Hence, the code of the read() operation, described in Figure 5, is a simplified version of the code of the join() operation.

Each read invocation is identified by a pair made up of the process index $i$ and a read sequence number read_sn $n_{i}$ (line 03). So, $p_{i}$ first broadcasts a read request $\operatorname{READ}\left(i, r e a d_{-} s n_{i}\right)$. Then, after it has received "enough" replies, $p_{i}$ selects the one with the greatest sequence number, updates (if needed) its local pair $\left(\right.$ register $\left._{i}, s n_{i}\right)$, and returns the value of register $_{i}$.

When it receives a message $\operatorname{READ}\left(j, r_{-} s n\right), p_{i}$ sends back a reply to $p_{j}$ if it is active (line 09$)$. If it is joining the system, it remembers that it will have to send back a reply to $p_{j}$ when it will terminate its join operation (line 10).

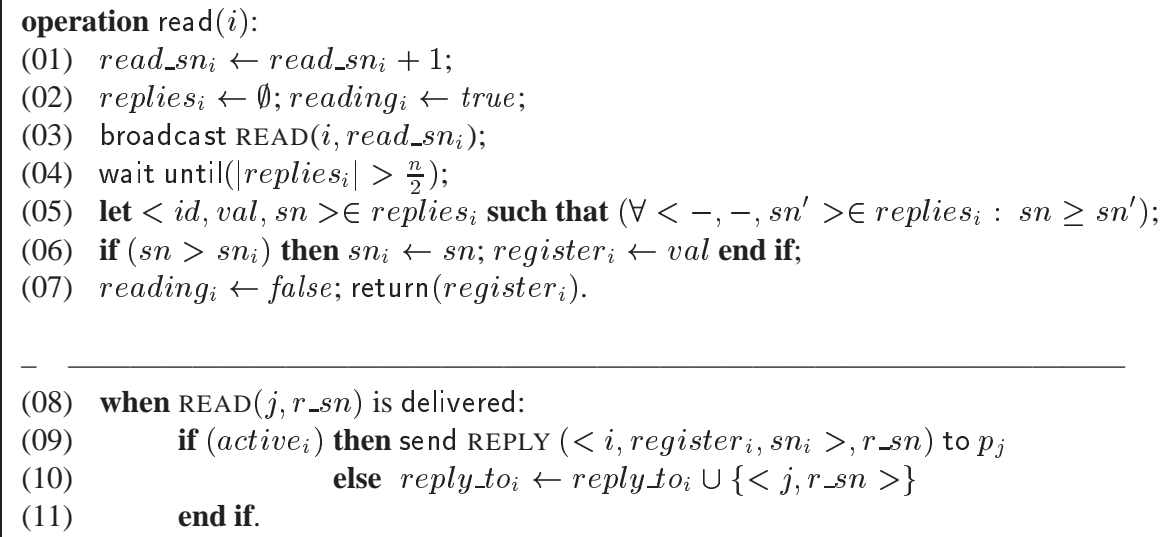

Figure 5: The read() protocol for an eventually synchronous system (code for $p$ )

The write() operation The code of the write operation is described in Figure 6. Let us recall that it is assumed that a single process at a time issues a write.

When a process $p_{i}$ wants to write, it issues first a read operation in order to obtain the sequence number associated with the last value written (line 01$)^{8}$. Then, after it has broadcast the message WRITE $(i,<$ $v, s n_{i}>$ ) to disseminate the new value and its sequence number to the other processes (line 04), $p$ waits until it has received "enough" acknowledgments. When this happens, it terminates the write operation by returning the control value $o k$ (line 05 ).

When it is delivered a message $\operatorname{WRITE}(j,<v a l, s n>), p_{i}$ takes into account the pair $(v a l, s n)$ if it is more uptodate than its current pair (line 07). In all cases, it sends back to the sender $p$ a message ACK $(i, s n)$ to allow it to terminate its write operation (line 08).

\footnotetext{
${ }^{7}$ As indicated before, the "read" identified $(i, 0)$ is the join () operation issued by $p_{i}$.

${ }^{8}$ As the write operations are not concurrent, this read obtains the greatest sequence number. The same strategy to obtain the last sequence number is used in protocols implementing an atomic registers (e.g., $[3,10])$.
}

PI $n{ }^{\circ} 1913$ 
When it receives a message $\operatorname{ACK}(j, s n), p_{i}$ adds it to its set write_ack $k_{i}$ if this message is an answer to its last write (line 10).

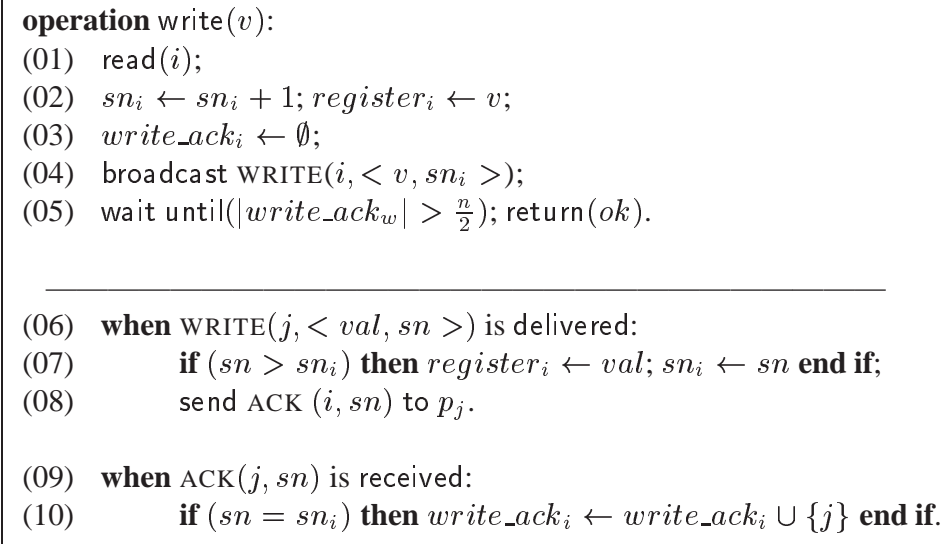

Figure 6: The write() protocol for an eventually synchronous system (code for $p$ )

\subsection{Proof of the eventually synchronous protocol}

Lemma 5 Let us assume that (1) $\forall \tau:|A(\tau)|>\frac{n}{2}$, and (2) a process that invokes the join() operation remains in the system for at least $3 \delta$ time units. If a process $p_{i}$ invokes the join() operation and does not leave the system, this join operation terminates.

Proof Let us first observe that, in order to terminate its join() operation, a process $p$ has to wait until its set replies $_{i}$ contains $\frac{n}{2}$ elements (line 04, Figure 4). Empty at the beginning of the join operation (line 01, Figure 4), this set is filled in by $p_{i}$ when it receives the corresponding REPLY () messages (line 20 of Figure 4).

A process $p_{j}$ sends a REPLY() message to $p_{i}$ if (i) either it is active and has received an INQUIRY message from $p_{i}$, (line 13, Figure 4), or (ii) it terminates its join() join operation and $<i,->\in r e p l y_{-} t o_{j} \cup d l_{\_} p r e v_{j}$ (lines 08-09, Figure 4).

Let us suppose by contradiction that $\mid$ replies $_{i} \mid$ remains smaller than $\frac{n}{2}$. This means that $p_{i}$ does not receive enough REPLY() carrying the appropriate sequence number. Let $\tau$ be the time at which the system becomes synchronous and let us consider a time $\tau^{\prime}>\tau$ at which a new process $p_{j}$ invokes the join operation. At time $\tau^{\prime}, p_{j}$ broadcasts an INQUIRY message (line 03, Figure 4). As the system is synchronous from time $\tau$, every process present in the system during $\left[\tau^{\prime}, \tau^{\prime}+\delta\right]$ receives such INQUIRY message by time $\tau^{\prime}+\delta$.

As it is not active yet when it receives $p_{j}$ 's INQUIRY message, the process $p_{i}$ executes line 16 of Figure 4 and sends back a DL_PREV message to $p_{j}$. Due to the assumption that every process that joins the system remains inside for at least $3 \delta$ time units, $p_{j}$ receives $p_{i}$ 's DL_PREV and executes consequently line 22 (Figure 4) adding $<i,->$ to $d l_{-}$prev $_{j}$.

Due to the assumption that there are always at least $\frac{n}{2}$ active processes in the system, we have that at time $\tau^{\prime}+\delta$ at least $\frac{n}{2}$ processes receive the INQUIRY message of $p_{j}$, and each of them will execute line 13 (Figure 4) and send a REPLY message to $p_{j}$. Due to the synchrony of the system, $p_{j}$ receives these messages by time $\tau^{\prime}+2 \delta$ and then stops waiting and becomes active (line 07, Figure 4). Consequently (lines 08-09) $p_{j}$ sends a REPLY to $p_{i}$ as $i \in$ reply_to $t_{j} \cup d l_{-} p r e v_{j}$. By $\delta$ time units, $p_{i}$ receives that REPLY message and executes line 20, Figure 4. Due to churn rate, there are an infinity of processes invoking the join after time $\tau$ 
and $p_{i}$ will receive a reply from any of them so $p_{i}$ will fill in its set replies $i$ and terminate its join operation.

Lemma 6 Let us assume that (1) $\forall \tau:|A(\tau)|>\frac{n}{2}$, and (2) a process that invokes the join() operation remains in the system for at least $3 \delta$ time units. If a process $p_{i}$ invokes a read () operation and does not leave the system, this read operation terminates.

Proof The proof of the read termination is the same as that of Lemma 5. In fact the read is a simplified case of the join algorithm in which the process $p_{i}$ that issues the operation is already active. Due top page limitation, the proof is left to the reader.

Lemma 7 Let us assume that (1) $\forall \tau:|A(\tau)|>\frac{n}{2}$, and (2) a process that invokes the join() operation remains in the system for at least $3 \delta$ time units. If a process invokes write() and does not leave, this write operation terminates.

Proof Let us first assume that the read() operation invoked at line 01 terminates (this is proved in Lemma 6 ). Before terminating the write of a value $v$ with a sequence number $s n b$ a process $p$ has to wait until its set write_ack $k_{i}$ contains at least $\frac{n}{2}$ elements (line 05, Figure 6). Empty at the beginning of the write operation (line 03), this set is filled in when the $\operatorname{ACK}(-, s n b)$ ) messages are delivered to $p_{i}$ (line 10). Such an ack message is sent by every process $p_{j}$ such that (i) $p_{j}$ receives the corresponding WRITE message from $p_{i}$ (line 08) or (ii) $p_{j}$ receives a REPLY message for its join from $p_{i}$ (line 20, Figure 4).

Suppose by contradiction that $p_{i}$ never fills in write_ack $k_{i}$. This means that $p_{i}$ misses ACK() messages carrying the sequence number $s n b$. Let us consider the time $\tau$ at which the system becomes synchronous, i.e., every message sent by any process $p_{j}$ at time $\tau^{\prime}>\tau$ is delivered by time $\tau^{\prime}+\delta$. Due to the assumption that the writer does not leave before the termination of its write, it follows that $p$ will receive all the INQUIRY messages sent by processes joining after time $\tau$. When it receives an INQUIRY() message from some joining process $p_{j}, p_{i}$ executes line 13 of Figure $4^{9}$ and sends a REPLY message to $p_{j}$ with the sequence number $s n b$. Since, after $\tau$, the system is synchronous, $p_{j}$ receives this REPLY message in at most $\delta$ time units and executes line 20 of Figure 4 sending back an $\operatorname{ACK}(-, s n b)$ message to $p_{i}$. As (1) by assumption a process that joins the system does not leave for at least $3 \delta$ time units and (2) the system is now synchronous, such an $\operatorname{ACK}(-, s n b)$ message is received by $p_{i}$ in at most $\delta$ time units and consequently $p_{i}$ executes line 10 and adds $p_{j}$ to the set write_ack $k_{i}$. Due to the dynamicity of the system (captured by the churn rate $c$ ), processes continuously join the system. Due to the chain of messages INQUIRY (), REPLY(), ACK(), the reception of each message triggering the sending of the next one, it follows that $p_{\imath}$ eventually receives $\frac{n}{2} \operatorname{ACK}(-, s n b)$ messages and terminates its write operation.

$\square_{\text {Lemma }} 7$

Theorem 3 Termination. Let us assume that $\forall \tau:|A(\tau)|>\frac{n}{2}$. If a process invokes join(), read() or write (), and does not leave the system, it terminates its operation.

Proof It follows from Lemma 5, Lemma 6 and Lemma 7.

Theorem 4 Safety. Let us assume that $\forall \tau:|A(\tau)|>\frac{n}{2}$. A read operation returns the last value written before the read invocation, or a value written by a write operation concurrent with it.

\footnotetext{
${ }^{9}$ The writer process $p_{i}$ executes line 13 of Figure 4 because a writer is always in the active mode.
} 
Proof (Sketch) Let write $(v)$ be the $\alpha$-th write operation invoked on the register, and $W_{\alpha}(\tau)$ the set of processes that, at time $\tau$, have the corresponding value $v$ in their local copy of the regular register (to simplify the reasoning, and without loss of generality, we assume that no two write operations write the same value).

Let $\tau_{0}$ be the starting time. It follows from the initialization statement, that the $n$ processes that initially define the system are active and contain the initial value of the regular register (say $\succcurlyeq$ ). Consequently, we have $\left|A\left(\tau_{0}\right)\right|=\left|W_{0}\left(\tau_{0}\right)\right|>\frac{n}{2}$.

Let $\tau_{y}=\tau_{0}+y$ (the time instant that is $y$ time units after $\tau_{0}$ ). By time $\tau_{1}$, nc processes leave the system and $n c$ processes invoke the join operation. All the processes that leave were active at time $\tau$ and their local copy of the register contained $v_{0}$. Hence, $\left|A\left(\tau_{1}\right)\right|>\frac{n}{2}$ and $\left|W_{0}\left(\tau_{1}\right)\right|>\frac{n}{2}$. As $\forall \tau:|A(\tau)|>$ $\frac{n}{2}$.(assumption), at most one process in $A\left(\tau_{1}\right)$ (and then also in $W_{0}\left(\tau_{1}\right)$ ) can leave before any process entering the system terminates its join operation.

Let $p_{i}$ be the first process that terminates its join operation. If no write operation is concurrent with that join, then all of the replies received by $p_{i}$ come from processes in $W_{0}\left(\tau_{1}\right)$. Each of these processes stores the last value written (namely, the initial value $w_{0}$ ) in its local copy of the register together with the sequence number 0 . When $p_{i}$ executes the lines $05-06$ of the join() operation (Figure 4), it updates its local variable with the value $v_{0}$.

If there is a concurrent write operation ( write $_{1}\left(v_{1}\right)$ ) issued by a process, it is possible that, before the end of this write, (1) active processes receive the WRITE message and consequently update their local copy of the register with $v_{1}$ (line 05, Figure 6), while (2) other active processes still keep the value $\vartheta_{0}$. At any $\tau$ between the invocation and the end of write $\left(v_{1}\right)$, we have $\left|W_{i}(\tau)\right|=x$ and $\left|W_{0}(\tau)\right|=|A(\tau)|-x$, where $x$ is the number of processes that, at time $\tau$, have updated to $v_{1}$ their local copy of the register. Due to the asynchrony of the system we cannot know when an active process replies an INQUIRY message. It can reply before receiving the WRITE message or after. If they all reply before, $p_{\imath}$ will return the last value written before the concurrent write write ${ }_{1}(v)$. Otherwise, $p_{i}$ will return a concurrently written value. Note that, in order to terminate (say at time $\tau_{E}$ ), the write ${ }_{1}\left(v_{1}\right)$ operation needs to receive $n(1-c)$ ACK () messages (line 03, Figure 6). Hence, we have $\left|W_{1}\left(\tau_{E}\right)\right| \geq \frac{n}{2}$. Then, by iterating the above reasoning, we have that any join concurrent with write ${ }_{1}\left(v_{1}\right)$ will return either the last written value or the value concurrently written, and any subsequent join will return the last written value.

Then, the reasoning is the same as before for the subsequent write operations. It follows that a read obtains the last value written it there is no concurrent write operations, or the last value written before the read invocation, or a value written by a write operation concurrent with it.

$\square$ Theorem 4

\section{Related Work}

Assumption of correct processes in the system [24] and [28] describe protocols implementing distributed computing abstractions on top of a dynamic distributed system (leader election and connectivity, respectively) together with their correctness proofs. These protocols assume that a certain number of processes remain in the system for "long enough" periods (as an example, [28] requires the perpetual presence of at least one process to maintain the system connectivity). The regular register protocols that have been presented do not require such an assumption (any process can be replaced at any time as allowed by the constant churn).

Registers implementation in mobile ad-hoc networks To the best of our knowledge the proposed regular register protocols are the firsts for distributed systems subject to churn. Other register protocols have been designed for mobile ad-hoc networks, e.g., [8]. Interestingly this protocol relies on a form of deterministic 
broadcast, namely, geocast which is similar to our broadcast primitive in the sense that it ensures delivery to the processes that stay long enough in the system without leaving. Register protocols for MANET have been provided for synchronous systems.

\section{Conclusion}

This paper has addressed the construction of a regular register in synchronous and eventually synchronous message-passing distributed systems where processes can join and leave the system. Two protocols have been presented and proved correct, one for each type of system.

Several questions remain unanswered. One concerns the churn rate $c$. If there is no constraint on $c$, there is no protocol implementing a shared register (intuitively, this is because it is possible that no process remains long enough in the system to be able to implement the permanence/durability associated with a register). So, a fundamental question is the following: Is it possible to characterize the greatest value of $c$ for a synchronous system (defined as a function involving the delay upper bound $\delta$ )? Moreover, has $c$ to depend on $n$ (the system size) in an asynchronous system? Another question concerns the implementation of quorums in dynamic systems ${ }^{10}$ (quorums are required if one wants to permit any process: to write at any time). Finally, another important question is how to cope with the additional adversary that are process failures in a dynamic system?

\section{References}

[1] Abraham, I. and Malkhi, D., Probabilistic Quorum Systems for Dynamic Systems. Distributed Computing, 18(2):113-124, 2005.

[2] Aguilera M.K., A Pleasant Stroll Through the Land of Infinitely Many Creatures. ACM SIGACT News, Distributed Computing Column, 35(2):36-59, 2004.

[3] Attiya H., Bar-Noy A. and Dolev D., Sharing Memory Robustly in Message-Passing Systems. Journal of the ACM, 42(1):129-142, 1995.

[4] Baldoni R., Bertier M., Raynal M. and Tucci S., Looking for a Definition of Dynamic Distributed Systems. Proc. 9th Int'l Conference on Parallel Computing Technologies (PaCT'07), Springer Verlag LNCS \#4671, pp. 1-14, 2007.

[5] Bloom B., Constructing Two-writer Atomic Registers. IEEE Transactions on Computers, 37:1506-1514, 1988.

[6] Chandra T. and Toueg S., Unreliable Failure Detectors for Reliable Distributed Systems. Journal of the ACM, 43(2):225-267, 1996.

[7] Chaudhuri S., Kosa M.J. and Welch J., One-write Olgorithms for Multivalued Regular and Atomic Registers. Acta Informatica, 37:161-192, 2000.

[8] Dolev S., Gilbert S., Lynch N., Shvartsman A., and Welch J., Geoquorum: Implementing Atomic Memory in Ad hoc Networks. Proc. 17th Int'l Symposium on Distributed Computing (DISC'03), Springer-Verlag LNCS \#2848, pp. 306-320, 2003.

[9] Dwork C., Lynch N. and Stockmeyer L., Consensus in the Presence of Partial Synchrony. Journal of the ACM, 35(2):288-323, April 1988.

\footnotetext{
${ }^{10}$ Dynamic quorums are addressed in a few papers, e.g., $[1,13,17,23,26]$.
} 
[10] Friedman R., Raynal M. and Travers C., Abstractions for Implementing Atomic Objects in Distributed Systems. 9th Int'l Conference on Principles of Distributed Systems (OPODIS'05), Springer Verlag LNCS \#3974, pp. 73-87, 2005.

[11] Gafni E. and Lamport L., Disk Paxos. Distributed Computing, 16(1):1-20, 2003.

[12] Gafni E., Merritt M., and Taubenfeld G., The Concurrency Hierarchy, and Algorithms for Unbounded Concurrency. Proc. 20th ACM Symposium on Principles of Distributed Computing (PODC'01), pp. 161-16, 2001.

[13] Gramoli V. and Raynal M., Timed Quorum Systems for Large-Scale and Dynamic Environments. Proc. 11th Int'l Conference on Principles of Distributed Systems (OPODIS'07), Springer-Verlag, LNCS \# 4878, pp. 429-442, 2007.

[14] Guerraoui R. and Raynal M., The Alpha of Indulgent Consensus. The Computer Journal, 50(1):53-67, 2007.

[15] Hadzilacos V. and Toueg S., Reliable Broadcast and Related Problems. In Distributed Systems, ACM Press, New-York, pp. 97-145, 1993.

[16] Haldar S. and Vidyasankar K., Constructing 1-writer Multireader Multivalued Atomic Variables from Regular Variables. Journal of the ACM, 42(1):186-203, 1995.

[17] Herlihy, M., Dynamic Quorum Adjustment for Partitioned Data. ACM Transactions on Database Systems (TODS), 12(2):170-194, 1987.

[18] Herlihy M.P. and Wing J.M., Linearizability: a Correctness Condition for Concurrent Objects. ACM Transactions on Programming Languages and Systems, 12(3):463-492, 1990.

[19] Ko S., Hoque I. and Gupta I., Using Tractable and Realistic Churn Models to Analyze Quiescence Behavior of Distributed Protocols. Proc. 27th IEEE Int'l Symposium on Reliable Distributed Systems (SRDS'08), IEEE Computer Press, pp. 259-268, 2008.

[20] Lamport. L., On Interprocess Communication, Part 1: Models, Part 2: Algorirhms. Distributed Computing, 1(2):77-101, 1986.

[21] Li M., Tromp J. and Vityani P., How to Share Concurrent Wait-free Variables. Journal of the ACM, 43(4):723746, 1996.

[22] Liben-Nowell D., Balakrishnan H., and Karger D.R., Analysis of the Evolution of Peer-to-peer Systems. Proc. 21th ACM Symposium on Principles of Distributed Computing (PODC'02), ACM press, pp 233-242, 2002.

[23] Lynch, N. and Shvartsman A., RAMBO: A Reconfigurable Atomic Memory Service for Dynamic Networks. Proc. 16th Int'l Symposium on Distributed Computing (DISC'02), Springer-Verlag LNCS \#2508, pp. 173-190, 2002.

[24] Mostefaoui A., Raynal M., Travers C., Peterson S., El Abbadi, Agrawal D., ¿From Static Distributed Systems to Dynamic Systems. 24th IEEE Symposium on Reliable Distributed Systems (SRDS'05), IEEE Computer Society Press, pp. 109-119, 2005.

[25] Merritt M. and Taubenfeld G., Computing with Infinitely Many Processes. Proc. 14th Int'l Symposium on Distributed Computing (DISC'00), Springer-Verlag LNCS \#1914, pp. 164-178, 2000.

[26] Nadav U. and Naor M., The Dynamic And-or Quorum System. Proc. 19th Int'l Symposium on Distributed Computing (DISC'05), Springer-Verlag LNCS \#3724, pp. 472-486, 2005.

[27] Singh A.K., Anderson J.H. and Gouda M., The Elusive Atomic Register. Journal of the ACM, 41(2):331-334, 1994.

[28] Tucci Piergiovanni S. and Baldoni R., Connectivity in Eventually Quiescent Dynamic Distributed Systems, Third Latin-American Symposium on Dependable Computing (LADC07), IEEE Press, pp. 38-56, 2007. 
[29] Vidyasankar K., Converting Lamport's Regular Register to Atomic Register. Information Processing Letters, 28(6):287-290, 1988

[30] Vityani P. and Awerbuch B., Atomic Shared Register Access by Asynchronous Hardware. Proc. 27th IEEE Symposium on Foundations of Computer Science (FOCS'87), IEEE Computer Press, 223-243, 1986.

PI ${ }^{\circ} 1913$ 